\title{
The Efficacy and Response Predictors of Platinum-Based Neoadjuvant Chemotherapy in Locally Advanced Cervical Cancer
}

This article was published in the following Dove Press journal: Cancer Management and Research

\author{
Yuhui Huang* \\ Lin Liu* \\ Jing Cai $(\mathbb{D}$ \\ Lu Yang \\ Si Sun \\ Jing Zhao \\ Zhoufang Xiong (ID \\ Zehua Wang $\mathbb{D}$
}

Department of Obstetrics and Gynecology, Union Hospital, Tongji Medical College, Huazhong University of Science and Technology, Wuhan 430022, Hubei, People's Republic of China

*These authors contributed equally to this work
Correspondence: Zehua Wang; Zhoufang Xiong

Department of Obstetrics and Gynecology, Union Hospital, Tongji Medical College, Huazhong University of Science and Technology, 1277 Jiefang Avenue, Wuhan 430022, Hubei, People's Republic of China

Tel/Fax +862785351649

Email zehuawang@।63.net;

xiaoxiongmaomi@aliyun.com
Objective: To assess the efficacy of platinum-based neoadjuvant chemotherapy (NACT) in patients with locally advanced cervical cancer (LACC) and investigate the pretreatment predictors of the response.

Patients and Methods: A total of 219 patients with International Federation of Gynecology and Obstetrics (FIGO 2009) stage IB2-IIA2 LACC who received platinumbased NACT from December 2007 to December 2017 were reviewed, and their clinicalpathological characteristics and follow-up data were retrospectively collected and analyzed. The baseline characteristics of age, FIGO stage, histology, tumor differentiation, tumor size, and clinical outcomes, including post-operative pathological risk factors, overall survival (OS), and progression-free survival (PFS) were compared between the responders and nonresponders.

Results: The overall response rate was 58.9\% (129/219), and 19 (8.7\%) patients achieved pathologically complete remission. NACT responders showed significantly better OS and PFS than non-responders $\left(\mathrm{P}_{\mathrm{OS}}=0.002, \mathrm{P}_{\mathrm{PFS}}=0.002\right)$. The response to NACT was identified as an independent risk factor for OS (hazard ratio $[\mathrm{HR}]=2.453,95 \%$ confidence intervals $[95 \% \mathrm{CI}], 1.125-5.348, P=0.024)$ and PFS $(\mathrm{HR}=2.196,95 \% \mathrm{CI}, 1.183-4.076, P=0.013)$, and patients with IB2/IIA1 and a tumor size of $<5 \mathrm{~cm}$ tended to receive better response than patients with IIA2 $(P=0.004)$ and a tumor size of $\geq 5 \mathrm{~cm}(P=0.027)$.

Conclusion: The response rate of platinum-based NACT was approximately $60 \%$. The response to NACT was an independent risk factor for prognosis, and patients with earlier stage and smaller tumor tended to respond better to NACT.

Keywords: locally advanced cervical cancer, neoadjuvant chemotherapy, clinical response, predictor, prognosis

\section{Introduction}

Cervical cancer is the fourth most common cancer in women worldwide with approximately 569,847 new cases and 311,365 deaths in 2018 alone, ${ }^{1}$ which makes it one of the leading causes of cancer death for women. Despite advances in the screening technology for cervical cancer, some patients remained diagnosed with locally advanced cervical cancer (LACC). Patients with LACC at presentation are at higher risk of recurrence and account for more cervical cancer deaths than earlier-stage patients, ${ }^{2}$ and an effective treatment for LACC remains an challenging task.

Concurrent chemoradiation is recommended as the standard treatment for LACC with an improved survival. ${ }^{3}$ However, radiation for the special anatomical 
position of the cervix sometimes leads to serious complications of gastrointestinal and genitourinary toxicity and impacts the quality of life. Neoadjuvant chemotherapy (NACT) followed by radical hysterectomy and pelvic lymph node resection is a promising strategy for LACC and exhibits favorable toxicity and comparable or improved survival, ${ }^{4}$ especially in developing countries where the access and technological development of radiation are limited.

The efficacy of NACT in patients with LACC remains controversial. The overall response rate to NACT ranged from $52 \%$ to $95 \%$ in different studies. ${ }^{5,6}$ For long-term survival, some studies showed that the response to NACT was an important prognostic factor, and NACT responders benefited from NACT with improved overall survival (OS) and progression-free survival (PFS) compared to nonresponders, ${ }^{7-10}$ which is consistent with our previous study. ${ }^{11}$ However, some other studies failed to identify an effect of response on survival in patients with LACC. ${ }^{12}$ Therefore, whether NACT responders benefit from NACT and which patients would have a tendency to respond to NACT remain controversial. In this study, we assessed the efficacy of NACT and aimed to investigate the effect of early response on prognosis and find pretreatment factors that may be able to predict the early response to NACT.

\section{Patients and Methods}

\section{Patients}

Patients with International Federation of Gynecology and Obstetrics (FIGO 2009) stage IB2-IIA2 cervical cancer who underwent $2-3$ cycles NACT before radical surgery or radiation from December 2007 to December 2017 in our hospital were included in this study.

The diagnoses of invasive cervical cancer were confirmed by cervix biopsy and pathological examination before treatment, and the staging was based on pelvic examination, Magnetic Resonance Imaging (MRI), and Computed Tomography (CT). Patients who received preoperative radiotherapy or suffered from other malignancies were excluded. Clinical and pathological data were collected from patients' medical records.

\section{Management}

All patients received 2-3 cycles of paclitaxel-carboplatin NACT. Patients intravenously received paclitaxel (paclitaxel liposome for injection, Luye Pharma Group Ltd., Nanjing, China) at $135-175 \mathrm{mg} / \mathrm{m}^{2}$ on the first day and platinum (carboplatin for injection, Qilu Pharma Ltd., China) at an area under the curve (AUC) $=5$ on the second day every 3 weeks.

Assessment of the response to NACT was based on the Response Evaluation Criteria In Solid Tumors (RECISR, version1.1), briefly, complete response (CR) was defined as disappearance of all target lesions, partial response (PR) was defined as at least a $30 \%$ decrease in the sum of diameters of target lesions, progressive disease (PD) was defined as at least a $20 \%$ increase in the sum of diameters of target lesions, and stable disease (SD) was defined as neither sufficient shrinkage to qualify for PR nor sufficient increase to qualify for PD. ${ }^{13}$ Responders (NACT-R) were defined as patients with $\mathrm{CR}$ or PR, and non-responders (NACT-NR) were defined as patients with SD or PD. Toxicity was defined according to the Common Terminology Criteria for Adverse Events (CTCAE, version 4.0).

After completion of NACT, the operable patients underwent radical surgery within 3 weeks. Patients who responded to NACT and those with IB2 tumor who showed stable disease but were deemed operable were also subjected to surgery. Adjuvant chemotherapy or radiotherapy was performed in patients with any highrisk factors (ie lymph node metastasis, parametrial involvement and positive surgical margins) and patients with two or more intermediate risk factors (ie LVSI, deep stromal invasion, and tumor size $\geq 2 \mathrm{~cm}$ ). For inoperable patients, radiation or chemoradiation was performed.

\section{Follow-Up}

Patients were followed up every 3 6 months in the first two years, every six months in the third year, and once a year in the following years. The examinations included gynecological examination, vaginal stump cytological examination, pelvic ultrasound, and chest X-ray. OS was defined as the time from completion of NACT to death from any cause or to the date of last contact. PFS was defined as the time from completion of NACT to the first appearance of progressive disease or to the date of last contact.

\section{Statistical Analysis}

SPSS 23.0 (IBM Corp., Armonk, NY, USA) was used for statistical analysis. The statistically significance of variables associated with response was assessed using the $\chi^{2}$ test or Fisher's exact test, as appropriate. The variables in univariate and multivariate analyses were screened using 
Cox regression model analysis. The OS and PFS curves were generated using the Kaplan-Meier method with GraphPad Prism 7.0 (GraphPad Software, lnc., San Diego, CA, USA), and Log rank test was used to assess the significance of the differences. P-value $<0.05$ was considered statistically significant.

\section{Ethical Statement}

The study was approved by the Ethics Committee of the Tongji Medical College, Huazhong University of Science and Technology (No. 2018S452), compliance with the Declaration of Helsinki. Written informed consent was obtained from all participants before any treatment. All patients' information was anonymous.

\section{Results}

\section{Response to NACT}

A total of 219 patients, aged 25 to 68 years (median age, 46 years), with stage IB2-IIA2 cervical cancer who met the inclusion criteria were included in the present study. Their clinical-pathological characteristics are listed in Table 1. All patients received platinum-based NACT, which was generally well tolerated, and hematologicalrelated toxicity was the most frequent severe (grade 3/4) side effects: leukopenia occurred in $11.4 \%$ patients, and neutropenia occurred in $19.2 \%$ patients. No chemotherapyrelated deaths were seen. CR was observed in 35 (16.0\%) patients, including $19(8.7 \%)$ cases of pathological CR, and PR was observed in 94 (42.9\%) patients. Therefore, the overall response rate was $58.9 \%$ (129/219). Nonresponders included 82 patients with SD and 8 patients with PD. After NACT, 197 (90.0\%) patients received radical surgery and 19 patients were subjected to radiation or concurrent chemoradiation. Three patients received no further treatment. Moreover, comparison of post-operative pathology features revealed that lymph node metastasis, lymph vascular space invasion (LVSI), and parametrial infiltration in the responders occurred significantly less frequently than the non-responders, but the rates of positive surgery margins were similar (Table 2).

\section{Response to NACT Independently Predicts Longer Survival}

To verify the impact of response to NACT on the survival of patients with LACC, we compared OS and PFS in the responders and non-responders. With a median follow-up time of 48 (range, 3-150) months, the 5-year OS and PFS
Table I Characteristics of the Patients with LACC Undergoing NACT ( $\mathrm{N}=219)$

\begin{tabular}{|c|c|}
\hline Characteristics & $\mathbf{N}(\%)$ \\
\hline \multicolumn{2}{|l|}{ Age (year) } \\
\hline Median (range) & $46(25 \sim 68)$ \\
\hline Mean \pm standard deviation & $46( \pm 7.5)$ \\
\hline \multicolumn{2}{|l|}{ Histology } \\
\hline Squamous cell carcinoma & $186(84.9)$ \\
\hline Adenocarcinoma & $20(9.1)$ \\
\hline Adenosquamous carcinoma & $10(4.6)$ \\
\hline Others & $3(1.4)$ \\
\hline \multicolumn{2}{|l|}{ Stage (FIGO 2009) } \\
\hline IB2 & $97(44.3)$ \\
\hline$\| A \mid$ & $19(8.7)$ \\
\hline IIA2 & $103(47.0)$ \\
\hline \multicolumn{2}{|l|}{ Tumor size before NACT $(\mathrm{cm})$} \\
\hline$<4.0$ & $17(7.8)$ \\
\hline $4.0 \sim 4.9$ & $99(45.2)$ \\
\hline $5.0 \sim 5.9$ & $66(30.1)$ \\
\hline$\geq 6.0$ & $37(16.9)$ \\
\hline \multicolumn{2}{|l|}{ Tumor size after NACT $(\mathrm{cm})$} \\
\hline$<4.0$ & $149(68.0)$ \\
\hline$\geq 4.0$ & $70(32.0)$ \\
\hline \multicolumn{2}{|l|}{ Histologic grade } \\
\hline GI & $18(8.2)$ \\
\hline G2 & $103(47.0)$ \\
\hline G3 & $59(26.9)$ \\
\hline Unknown & $39(17.8)$ \\
\hline \multicolumn{2}{|l|}{ Lymph node metastasis } \\
\hline Negative & $135(61.6)$ \\
\hline Positive & $63(28.8)$ \\
\hline Unknown & $21(9.6)$ \\
\hline \multicolumn{2}{|l|}{ LVSI } \\
\hline Negative & $150(68.5)$ \\
\hline Positive & $47(21.5)$ \\
\hline Unknown & $22(10.1)$ \\
\hline \multicolumn{2}{|l|}{ Parametrial involvement } \\
\hline Negative & $169(77.2)$ \\
\hline Positive & $28(12.8)$ \\
\hline Unknown & $22(10.1)$ \\
\hline \multicolumn{2}{|l|}{ Surgical margin } \\
\hline Negative & $190(86.8)$ \\
\hline Positive & $7(3.2)$ \\
\hline Not applicable & $22(10.1)$ \\
\hline \multicolumn{2}{|l|}{ Response to NACT } \\
\hline CR & $35(16.0)$ \\
\hline PR & $94(42.9)$ \\
\hline SD & $82(37.4)$ \\
\hline PD & $8(3.7)$ \\
\hline
\end{tabular}

(Continued) 
Table I (Continued).

\begin{tabular}{|l|l|}
\hline Characteristics & N (\%) \\
\hline Therapy after NACT & \\
Radical surgery & $197(90.0)$ \\
Radiation or chemoradiation & $19(8.7)$ \\
No further treatment & $3(1.4)$ \\
\hline
\end{tabular}

Abbreviations: NACT, neoadjuvant chemotherapy; FIGO, International Federation of Gynecology and Obstetrics; LVSI, lymph vascular space invasion; $\mathrm{CR}$, complete response; $\mathrm{PR}$, partial response; SD, stable disease; PD, progressive disease.

Table 2 The Post-Operative Pathology in Patients with LACC Received NACT Before Surgery ( $\mathrm{N}=197)$

\begin{tabular}{|c|c|c|c|}
\hline & $\begin{array}{l}\text { Responders } \\
\text { N (\%) }\end{array}$ & $\begin{array}{l}\text { Non- } \\
\text { Responders } \\
\text { N (\%) }\end{array}$ & $\begin{array}{l}\text { P-value } \\
(\chi 2 \\
\text { Test) }\end{array}$ \\
\hline Total & $123(62.4)$ & 74 (37.6) & \\
\hline $\begin{array}{l}\text { Lymph node metastasis } \\
\text { Negative } \\
\text { Positive }\end{array}$ & $\begin{array}{l}93(75.6) \\
30(24.4)\end{array}$ & $\begin{array}{l}42(56.8) \\
32(43.2)\end{array}$ & 0.007 \\
\hline $\begin{array}{l}\text { LVSI } \\
\text { Negative } \\
\text { Positive }\end{array}$ & $\begin{array}{l}102(82.9) \\
21(17.1)\end{array}$ & $\begin{array}{l}48(64.9) \\
26(35.1)\end{array}$ & 0.006 \\
\hline $\begin{array}{l}\text { Parametrial involvement } \\
\text { Negative } \\
\text { Positive }\end{array}$ & $\begin{array}{l}\text { III (90.2) } \\
\text { I2(9.8) }\end{array}$ & $\begin{array}{l}58(78.4) \\
16(21.6)\end{array}$ & 0.034 \\
\hline $\begin{array}{l}\text { Surgery margin } \\
\text { Negative } \\
\text { Positive }\end{array}$ & $\begin{array}{l}118(95.9) \\
5(4.1)\end{array}$ & $\begin{array}{l}72(97.3) \\
2(2.7)\end{array}$ & 0.713 \\
\hline
\end{tabular}

Abbreviations: NACT, neoadjuvant chemotherapy; LVSI, lymph vascular space invasion.

in the responders $(85.4 \%$ and $71.4 \%$, respectively) were significantly higher than the non-responders $(63.3 \%$ and $52.4 \% ; \mathrm{P}_{\mathrm{OS}}=0.002, \mathrm{P}_{\mathrm{PFS}}=0.002$; Figure $1 \mathrm{~A}$ and $\left.\mathrm{B}\right)$. The subgroup analysis revealed that OS was not significantly different between the CR group and PR group, but the CR group and PR group had better OS than the nonresponders (Figure 1C). The PFS in patients with $\mathrm{CR}$ was significantly higher than the PR group and nonresponders (Figure 1D). To determine whether the response to NACT was an independent predictive factor for prognosis, univariate and multivariate analysis, including potential risk factors for survival, were performed. In the univariate analysis, the response to NACT, FIGO stage, lymph node metastasis, and LVSI were identified as risk factors for OS, and the response to NACT, FIGO stage, primary tumor $\geq 6 \mathrm{~cm}$, lymph node metastasis, LVSI, and parametrial involvement were identified as risk factors for PFS. In the multivariate analysis, the response to NACT remained significant for the OS and PFS. The nonresponders had a 2.453-fold increased risk of death and a 2.196-fold increased risk of disease progression (Table 3).

\section{Predictive Factors for the Response to NACT}

As the response to NACT is an important determinant for the survival of patients, the identification of predictive factors is meaningful. Correlations between NACT response and pre-NACT variables, including age, tumor stage, pathological type, histological grading, and primary tumor size, were evaluated (Table 4). Although the overall response rate was not significantly different between patients grouped by the above-mentioned characteristics, the $\mathrm{CR}$ rate was significantly increased in the tumors of earlier stage and smaller size. CR was achieved in $23.3 \%$ of the patients with IB2 and IIA1 and in $20.7 \%$ of patients with tumors smaller than $5 \mathrm{~cm}$, but only in $7.8 \%$ of patients with IIA2 tumors and in $10.7 \%$ of patients with larger tumors.

\section{Discussion}

The applicability of NACT in patients with LACC is debatable. Therefore, it is important to evaluate the efficacy of NACT in patients with LACC and select appropriate patients who may benefit from NACT. Our results showed that the overall response to NACT was $58.9 \%$, and the toxicity was mild. NACT responders significantly benefited from NACT with improved survival, and patients with early stage of IB2-IIA1 and small tumors $<5 \mathrm{~cm}$ tended to achieve a better response to NACT. These findings have important implications in improving the management of LACC by selecting the appropriate patients to receive NACT, and help non-responders avoid unnecessary chemotherapy-related toxicity and treatment delay.

Though concurrent chemoradiation is recommended as the standard treatment for patients with LACC, its complications seriously reduce the patients' quality of life. NACT followed by radical surgery is an alternative for the treatment of LACC with fewer complications and a favorable toxicity profile. The possible reasons supporting NACT may be that NACT can reduce tumor volume, improve the rate of tumor resection, as well as control the potential micro-metastasis. 

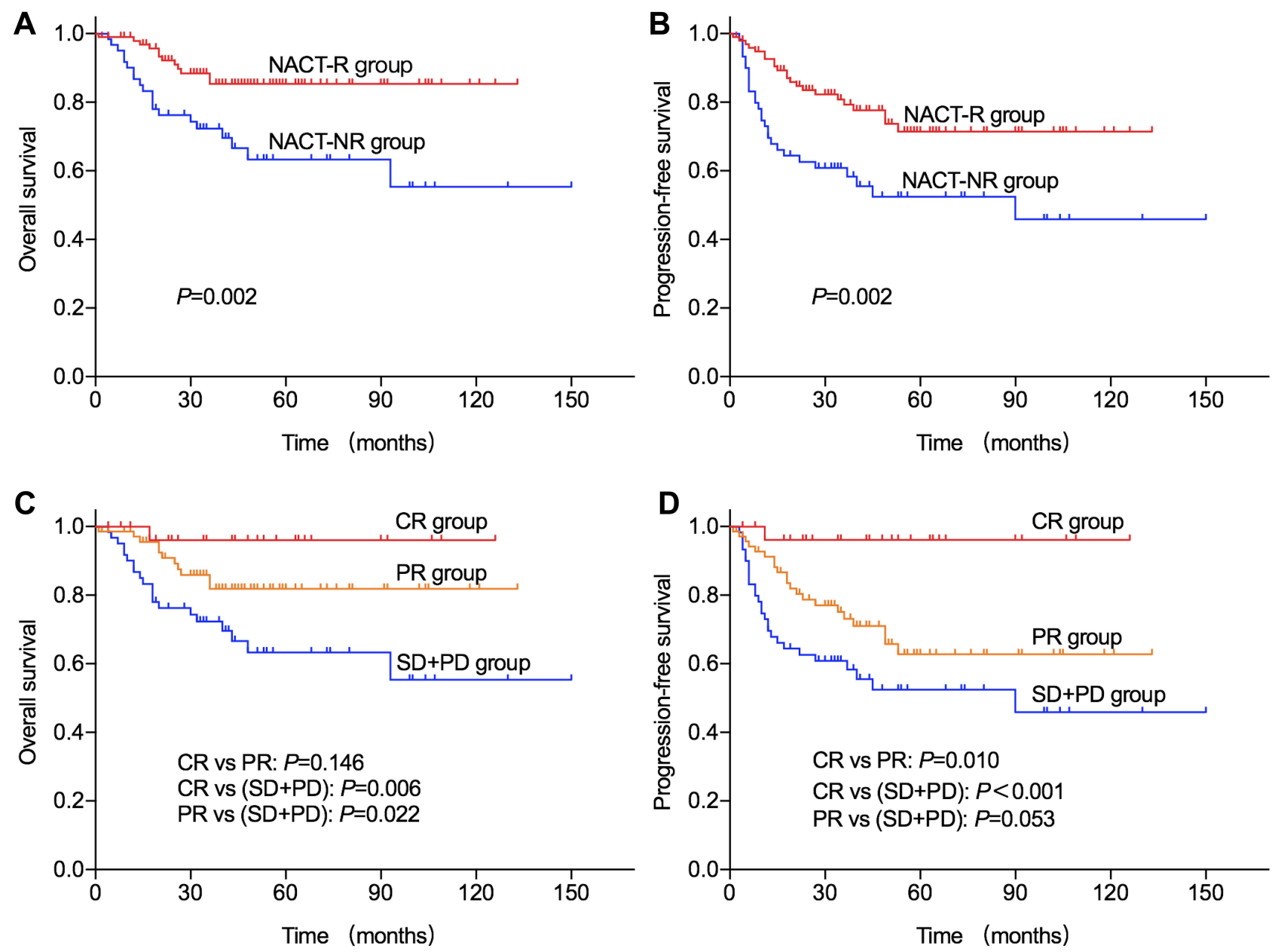

Figure I Correlation of response to NACT with OS and PFS in patients with LACC.

Notes: Kaplan-Meier survival curves are shown for OS (A) and PFS (B) in responders and non-responders, and OS (C) and PFS (D) in the subgroups of CR, PR, and nonresponders (SD+PD). Log rank test was used to assess the significance of the differences.

Abbreviations: NACT-R, neoadjuvant chemotherapy responders; NACT-NR, neoadjuvant chemotherapy non-responders; CR, complete response; PR, partial response; $\mathrm{SD}$, stable disease; PD, progressive disease; OS, overall survival; PFS, progression-free survival.

The response rate of NACT varied greatly in different studies. The overall response to NACT of paclitaxel and carboplatin in a Phase II study was $95 \%$, with $9 \%$ CR and $86 \% \mathrm{PR}^{6}{ }^{6}$ Huang $\mathrm{HJ}$ reported an overall response rate of 79.4\% after NACT with cisplatin, vincristine, and bleomycin in patients with bulky stage IB and IIA. ${ }^{14}$ Another retrospective study of patients with IB2-IIB showed a response rate of $65.9 \%$ at the end of NACT. ${ }^{15}$ The response rate in the present study was $58.9 \%$, which is lower than the response rate of $71.3 \%$ in our previous study. This difference may be explained by the higher proportion of patients with tumors $\geq 4 \mathrm{~cm}$ in the present study. Several other factors may also affect the response to NACT. Marita A. reported that the overall response rate with paclitaxel + carboplatin (PC) was significantly superior to topotecan + cisplatin (TC), and 3 4 cycles of NACT was more appropriate than $1 \sim 2$ cycles. ${ }^{16}$ Junker P. performed a small cohort study and found that the combination of a bevacizumab and platinum-containing chemotherapy improved the response rate $(42.9 \%$ vs $12.5 \%$ ) and the reduction in tumor size ( 3.7 vs $2.5 \mathrm{~cm}$ ) compared to a bevacizumab-free regimen after NACT in patients with FIGO stage Ib1 to IV. ${ }^{17}$ In patients with recurrent, persistent or metastatic cervical cancer, the addition of bevacizumab to chemotherapy was also associated with higher response rates than chemotherapy alone $(48 \%$ vs $36 \%) .{ }^{18}$ Studies also demonstrated that the route of administration of NACT affected the response to NACT. He Y. observed significantly higher response rates in the artery intervention group (97.8\%) compared to patients who received NACT via an intravenous route $(75.1 \%){ }^{7}$ Zhao et al made a similar discovery. ${ }^{19}$ In addition, the size 
Table 3 Univariate and Multivariate Analysis of Risk Factors for OS and PFS in LACC Patients Undergoing NACT

\begin{tabular}{|c|c|c|c|c|}
\hline \multirow[t]{2}{*}{ Variables } & \multicolumn{2}{|l|}{ Univariate } & \multicolumn{2}{|l|}{ Multivariate } \\
\hline & HR $(95 \% \mathrm{CI})$ & P-value & HR $(95 \% \mathrm{Cl})$ & P-value \\
\hline \multicolumn{5}{|l|}{ os } \\
\hline Response to NACT (no vs yes) & $3.250(1.544-6.838)$ & 0.002 & $2.453(1.125-5.348)$ & 0.024 \\
\hline Age $(\geq 45$ y vs $<45$ y) & $\mathrm{I} .193(0.558-2.55 \mathrm{I})$ & 0.649 & - & - \\
\hline Primary tumor size $(\geq 5 \mathrm{~cm}$ vs $<5 \mathrm{~cm}$ ) & $1.741(0.843-3.593)$ & 0.134 & - & - \\
\hline Primary tumor size ( $\geq 6 \mathrm{~cm}$ vs $<6 \mathrm{~cm}$ ) & $1.789(0.767-4.176)$ & 0.179 & - & - \\
\hline Histology (others vs squamous) & $2.048(0.910-4.609)$ & 0.083 & - & - \\
\hline FIGO stage (IIAI-IIA2 vs IB2) & $2.233(1.022-4.876)$ & 0.044 & $2.046(0.933-4.490)$ & 0.074 \\
\hline Histologic grade (G3 vs GI-G2) & $1.357(0.623-2.957)$ & 0.442 & - & - \\
\hline Lymph node metastasis (positive vs negative) & $2.572(1.253-5.280)$ & 0.010 & $1.652(0.711-3.839)$ & 0.243 \\
\hline LVSI (positive vs negative) & $2.560(1.229-5.334)$ & 0.012 & $1.686(0.710-4.006)$ & 0.237 \\
\hline Parametrial involvement (positive vs negative) & $1.979(0.806-4.860)$ & 0.137 & - & - \\
\hline Surgical margin (positive vs negative) & $1.874(0.255-13.794)$ & 0.538 & - & - \\
\hline \multicolumn{5}{|l|}{ PFS } \\
\hline Response to NACT (no vs yes) & $2.583(1.452-4.596)$ & 0.001 & $2.196(1.183-4.076)$ & 0.013 \\
\hline Age $(\geq 45$ y vs $<45$ y) & $0.728(0.408-1.299)$ & 0.283 & - & - \\
\hline Primary tumor size ( $\geq 5 \mathrm{~cm}$ vs $<5 \mathrm{~cm})$ & $1.700(0.956-3.025)$ & 0.071 & - & - \\
\hline Primary tumor size ( $\geq 6 \mathrm{~cm}$ vs $<6 \mathrm{~cm}$ ) & $2.019(1.026-3.973)$ & 0.042 & $2.013(0.995-4.07 I)$ & 0.052 \\
\hline Histology (others vs squamous) & $1.276(0.616-2.641)$ & 0.512 & - & - \\
\hline FIGO stage (IIAI-IIA2 vs IB2) & $1.902(1.040-3.479)$ & 0.037 & $1.600(0.868-2.947)$ & 0.132 \\
\hline Histologic grade (G3 vs GI-G2) & $0.874(0.460-1.660)$ & 0.680 & - & - \\
\hline Lymph node metastasis (positive vs negative) & $2.120(1.180-3.809)$ & 0.012 & $\mathrm{I} .098(0.53 \mathrm{I}-2.269)$ & 0.801 \\
\hline LVSI (positive vs negative) & $2.344(1.290-4.26 I)$ & 0.005 & $1.392(0.632-3.063)$ & $0.4 \mathrm{II}$ \\
\hline Parametrial involvement (positive vs negative) & $2.703(1.365-5.353)$ & 0.004 & $1.688(0.700-4.068)$ & 0.243 \\
\hline Surgical margin (positive vs negative) & $2.236(0.54 I-9.238)$ & 0.266 & - & - \\
\hline
\end{tabular}

Abbreviations: HR, hazard ratio; $\mathrm{Cl}$, confidence interval; FIGO, International Federation of Gynecology and Obstetrics; LVSI, lymph vascular space invasion; OS, overall survival; PFS, progression-free survival.

of trials and the inclusion of different patients in the studies may also affect the response, and large trials are needed to investigate the best strategy for the NACT.

The response to NACT is reported as one of the most vital prognostic factors for patients with LACC. Chen $\mathrm{H}$. demonstrated that responders had significantly longer PFS than non-responders, and the response was an independent prognostic predictor, with an hazard ratio (HR) of $6.963 .{ }^{20}$ Another phase II clinical study with NACT of CPT-11 and cisplatin revealed the responders had significantly lower risk of death than non-responders, with an HR of $0.025 .{ }^{21} \mathrm{Li}$ L. reported in their study that the responders had significant better OS and PFS than nonresponders with an $\mathrm{HR}=0.6$ for $\mathrm{OS}$ and $\mathrm{HR}=0.5$ for PFS after administration of TP, TC, or PF protocols in LACC. ${ }^{15}$ Some scholars also found that patients who did not achieve an overall optimal response had a higher risk of recurrence and death compared to patients who achieved optimal response. ${ }^{9}$ Our results revealed that the response to NACT was an independent prognostic factor, and the non-responders had an increased risk of 2.453-fold for death and 2.196-fold for disease progression. The difference in HR may be related to different response rates and the inclusion of patients. These results suggest that the response to NACT is an independent risk factor for longterm survival in patients with LACC and it is critical to select patients who potentially respond to NACT to give the therapy.

As responders may benefit from NACT with improved survival, non-responders would take unnecessary treatment and may end up with worse prognosis, the search for pretreatment predictive factors for response is important for the personalized treatment in LACC. In a previous study, patients with squamous cell tumors and patients with tumors $\leq 5 \mathrm{~cm}$ showed significantly higher response rates than patients with adenocarcinoma and tumors $>5 \mathrm{~cm} .{ }^{22}$ Huang $\mathrm{HJ}$ also discovered that patients with tumor $\leq 5 \mathrm{~cm}$ had significantly higher response rate than 
Table 4 Predictive Factors for the Response to NACT in Patients with LACC

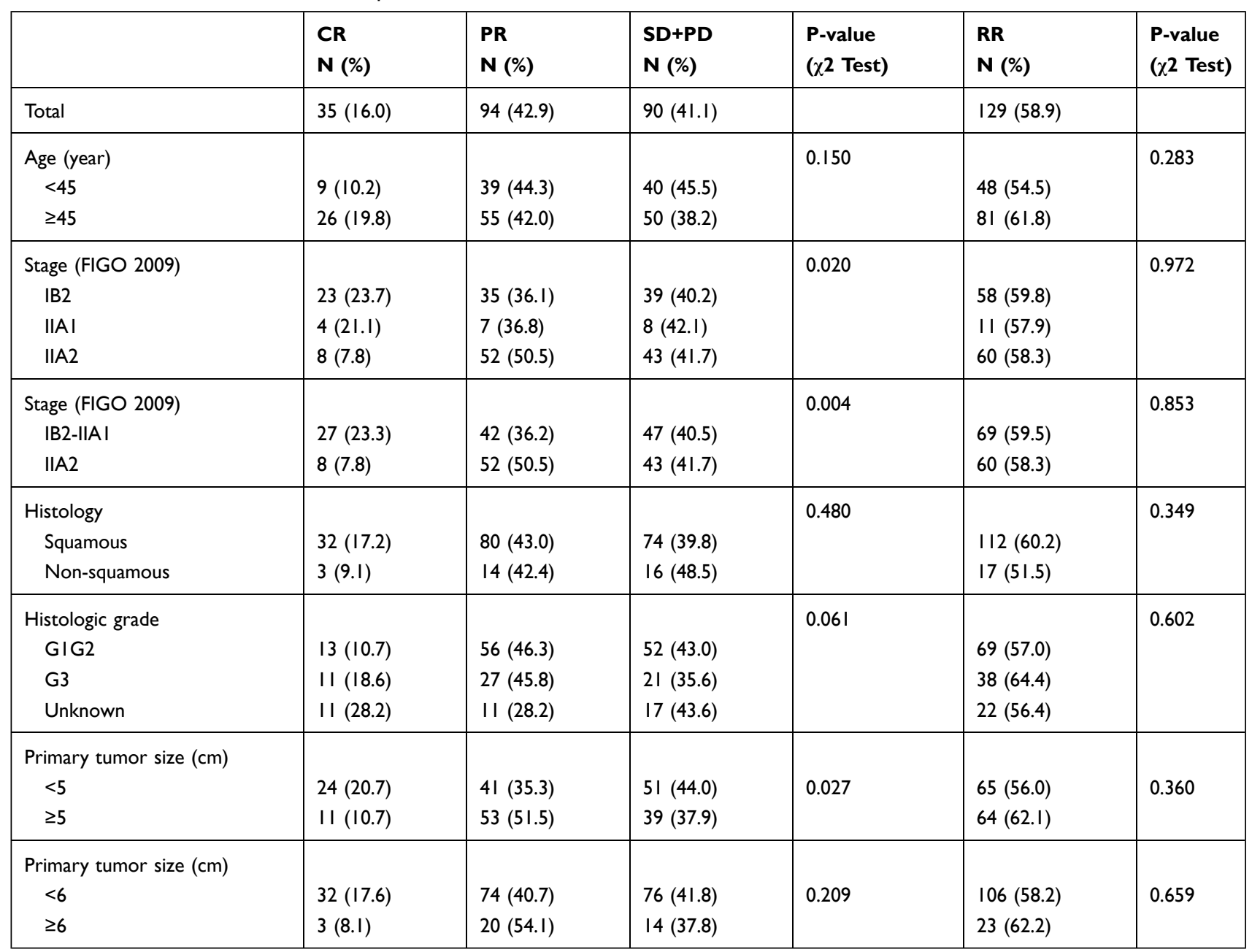

Abbreviations: CR, complete response; PR, partial response; SD, stable disease; PD, progression disease; RR, response rate; FIGO, International Federation of Gynecology and Obstetrics.

patients with tumor $>5 \mathrm{~cm}$, and patients older than 35 years may achieve a better response than younger patients. ${ }^{14}$ A randomized study had revealed that patients with squamous tumors and smaller tumors $<8 \mathrm{~cm}$ would receive a better response than patients with non-squamous and larger tumors after the administration of cisplatin, mitomycin, and 5-fluorouracil in patients with IB2-IIB. ${ }^{20}$ The present study observed that patients with earlier stage (IB2/IIA1) and smaller tumors (tumor with a size of $<5 \mathrm{~cm}$ ) achieved significantly more $\mathrm{CR}$ than other patients, which is consistent with previous studies. These results suggest that patients with earlier stage and smaller tumor more likely benefit from NACT, which should be verified in large scale, randomized studies.

We clarified the response rate and survival rate in a relatively large cohort of LACC patients undergoing carboplatin-paclitaxel NACT. However, the present study has several limitations. First, it is a non-randomized, retrospective study of single-center data; selection bias and confounding bias are inevitable. Second, the efficacy of NACT in terms of PFS and OS was not proven in the present of a control group, such as LACC patients undergoing primary chemoradiation. Third, all the patients studied received carboplatin-paclitaxel NACT, limiting generalization of the results. Fourth, the follow-up time for most patients was shorter than 60 months. Thus, further multi-center, randomized trials are needed to verify the value of NACT in patients with LACC.

\section{Conclusion}

In conclusion, our results suggested that the response to platinum-based NACT was approximately $60 \%$. The response to NACT was an independent risk factor for the survival of patients with LACC, and patients with earlier 
stage and smaller tumors had a tendency to receive a better response. These results provide important evidence in improving the management of LACC.

\section{Funding}

This work was supported by the National Natural Science Foundation of China (81974413).

\section{Disclosure}

The authors declare no conflicts of interest in this work.

\section{References}

1. Bray F, Ferlay J, Soerjomataram I, Siegel RL, Torre LA, Jemal A. Global cancer statistics 2018: GLOBOCAN estimates of incidence and mortality worldwide for 36 cancers in 185 countries. CA Cancer J Clin. 2018;68(6):394-424. doi:10.3322/caac.21492

2. Trattner M, Graf A-H, Lax S, et al. Prognostic factors in surgically treated stage $\mathrm{Ib}-\mathrm{IIb}$ cervical carcinomas with special emphasis on the importance of tumor volume. Gynecol Oncol. 2001;82(1):11-16. doi:10.1006/gyno.2001.6252

3. Koh W-J, Abu-Rustum NR, Bean S, et al. Cervical cancer, version 3.2019, NCCN clinical practice guidelines in oncology. J Natl Compr Canc Netw. 2019;17(1):64-84. doi:10.6004/jncen.2019.0001

4. Angioli R, Plotti F, Montera R, et al. Neoadjuvant chemotherapy plus radical surgery followed by chemotherapy in locally advanced cervical cancer. Gynecol Oncol. 2012;127(2):290-296. doi:10.1016/j.ygyno.20 12.07.104

5. Eddy GL, Bundy BN, Creasman WT, et al. Treatment of ("bulky") stage IB cervical cancer with or without neoadjuvant vincristine and cisplatin prior to radical hysterectomy and pelvic/para-aortic lymphadenectomy: A Phase III trial of the gynecologic oncology group. Gynecol Oncol. 2007;106(2):362-369. doi:10.1016/j.ygyno.2007. 04.007

6. Dueñas-Gonzalez A, López-Graniel C, González-Enciso A, et al. A phase II study of multimodality treatment for locally advanced cervical cancer: neoadjuvant carboplatin and paclitaxel followed by radical hysterectomy and adjuvant cisplatin chemoradiation. Ann Oncol. 2003;14(8):1278-1284. doi:10.1093/annonc/mdg333

7. He Y, Zhao Q, Geng Y, et al. Analysis of short-term efficacy as defined by RECIST and pathological response of neoadjuvant chemotherapy comprised paclitaxel and cisplatin followed by radical surgery in patients with locally advanced cervical cancer. Medicine. 2018;97 (22):e10913e10916. doi:10.1097/MD.0000000000010913

8. Zanaboni F, Grijuela B, Giudici S, et al. Weekly topotecan and cisplatin (TOPOCIS) as neo-adjuvant chemotherapy for locally-advanced squamous cervical carcinoma: results of a phase II multicentric study. Eur $J$ Cancer. 2013;49(5):1065-1072. doi:10.1016/j. ejca.2012.10.008

9. Xiong Y, Liang L, Cao L, Zheng M, Liu J. Clinical effects of irinotecan hydrochloride in combination with cisplatin as neoadjuvant chemotherapy in locally advanced cervical cancer. Gynecol Oncol. 2011;123(1):99-104. doi:10.1016/j.ygyno.2011.06.011
10. Gadducci A, Sartori E, Maggino T, et al. Pathological response on surgical samples is an independent prognostic variable for patients with Stage Ib2-IIb cervical cancer treated with neoadjuvant chemotherapy and radical hysterectomy: an Italian multicenter retrospective study (CTF Study). Gynecol Oncol. 2013;131(3):640-644. doi:10.1016/j.ygyno.2013.09.029

11. Yang L, Guo J, Shen Y, et al. Clinical efficacy and safety of paclitaxel plus carboplatin as neoadjuvant chemotherapy prior to radical hysterectomy and pelvic lymphadenectomy for Stage IB2-IIB cervical cancer. Int J Clin Exp Med. 2015;8:13690-13698.

12. Liu S, Yang J, Cao D, Shen K, Xiang Y, Lang J. Efficacy of neoadjuvant cisplatin and 5-flourouracil prior to surgery in FIGO stage IB2/IIA2 cervical cancer. Mol Clin Oncol. 2014;2(2):240-244. doi:10.3892/mco.2013.227

13. Eisenhauer EA, Therasse P, Bogaerts J, et al. New response evaluation criteria in solid tumours: revised RECIST guideline (version 1.1). Eur $J$ Cancer. 2009;45(2):228-247. doi:10.1016/j.ejca.20 08.10 .026

14. Huang HJ, Chang TC, Hong JH, et al. Prognostic value of age and histologic type in neoadjuvant chemotherapy plus radical surgery for bulky $(\geq 4 \mathrm{~cm})$ stage IB and IIA cervical carcinoma. Int J Gynecol Cancer. 2003;13:204-211.

15. Li L, Wu M, Ma S, Tan X, Zhong S. Neoadjuvant chemotherapy followed by radical hysterectomy for stage IB2-to-IIB cervical cancer: a retrospective cohort study. Int J Clin Oncol. 2019;24 (11):1440-1448. doi:10.1007/s10147-019-01510-1

16. Marita A, Ordeanu C, Rancea A, Nicolae T, Nagy VM. Long-term survival following neoadjuvant chemotherapy and concomitant radiochemotherapy in locally advanced cervical cancer: results of the oncology institute "Prof. Dr. Ion Chiricuta" experience. $J$ Med Life. 2018;11:42-50.

17. Junker P, Puppe J, Thangarajah F, et al. Neoadjuvant therapy of cervical carcinoma with the angiogenesis inhibitor bevacizumab: a single-centre analysis. Geburtshilfe Frauenheilkd. 2018;78(08): 768-774. doi:10.1055/a-0641-5588

18. Tewari KS, Sill MW, Long HJ, et al. Improved survival with bevacizumab in advanced cervical cancer. $N$ Engl J Med. 2014;370 (8):734-743. doi:10.1056/NEJMoa1309748

19. Zhao H, He Y, Zhu L, et al. Effect of neoadjuvant chemotherapy followed by radical surgery for FIGO stage IB2/IIA2 cervical cancer. Medicine. 2019;98(21):e15604. doi:10.1097/MD.0000000000015604

20. Chen H, Liang C, Zhang L, Huang S, Wu X. Clinical efficacy of modified preoperative neoadjuvant chemotherapy in the treatment of locally advanced (stage IB2 to IIB) cervical cancer: A randomized study. Gynecol Oncol. 2008;110(3):308-315. doi:10.1016/j.ygyno.20 08.05.026

21. Takatori E, Shoji T, Omi H, et al. Analysis of prognostic factors for patients with bulky squamous cell carcinoma of the uterine cervix who underwent neoadjuvant chemotherapy followed by radical hysterectomy. Int J Clin Oncol. 2014;20(2):345-350. doi:10.1007/ s10147-014-0702-6

22. Benedetti-Panici P, Greggi S, Scambia G, et al. Long-term survival following neoadjuvant chemotherapy and radical surgery in locally advanced cervical cancer. Eur J Cancer. 1998;34:341-346. doi:10.1016/S0959-8049(97)10029-6 


\section{Publish your work in this journal}

Cancer Management and Research is an international, peer-reviewed open access journal focusing on cancer research and the optimal use of preventative and integrated treatment interventions to achieve improved outcomes, enhanced survival and quality of life for the cancer patient.
The manuscript management system is completely online and includes a very quick and fair peer-review system, which is all easy to use. Visit http://www.dovepress.com/testimonials.php to read real quotes from published authors. 